\title{
AC losses of no-insulation high temperature superconductor (RE)Ba2Cu3Ox coils induced by ripple magnetic fields in machines
}

Yawei Wang, ${ }^{1,3)}$ Min Zhang, ${ }^{1)}$ Ján Šouc, ${ }^{2)}$ Fedor Gömöry, ${ }^{2)}$ Fangjing Weng, ${ }^{1)}$ Jianwei Li, ${ }^{4)}$ and Weijia Yuan ${ }^{1)}$

${ }^{1}$ Department of Electronic and Electrical Engineering, University of Strathclyde, Glasgow, G1 1XQ, UK

${ }^{2}$ Institute of Electrical Engineering, Slovak Academy of Sciences, Bratislava, Slovakia

${ }^{3}$ School of Electronic, information and Electrical Engineering, Shanghai Jiao Tong University, Shanghai, China

${ }^{4}$ National Engineering Science, University of Oxford, OX1 3PJ, Oxford, UK

\begin{abstract}
No-insulation high temperature superconductor (NI HTS) (RE)Ba2Cu3Ox coil technology is effective in enhancing the thermal stability of HTS coils. Applying the NI technique on the rotor windings of HTS machines can improve the stability and reliability of the machines. However, the NI HTS rotor windings experience ripple magnetic fields, which leads to induced eddy currents through the turn-to-turn contacts. The accompanying turn-to-turn losses will considerably affect the machine efficiency. In this study, we compared experimentally the losses of NI HTS coils subjected to external AC magnetic fields with those of insulated coils. Measurement system based on calibrationfree method is developed for the AC loss measurement on HTS coils exposed to external AC magnetic fields. The results show that the AC loss of NI HTS coils can be 20 times higher than that of insulated HTS coils, and lowering turn-to-turn resistivity can significantly reduce this AC loss. Modeling analysis shows that most of induced current flows in the outer turns of the NI HTS coil because of skin effect, and lower turn-to-turn resistivity leads to higher induced current in superconducting layers and more significant accumulation of turn-to-turn loss. This will increase quench risk. Optimization of turn-to-turn resistivity is required when the NI HTS coil is applied in the machines environments.
\end{abstract}

\section{Introduction}

The high temperature superconductor (HTS) (RE) $\mathrm{Ba}_{2} \mathrm{Cu}_{3} \mathrm{O}_{x}$ (REBCO) is a widely applied on many industrial applications because of its high current density and cost reduction potential[1-6]. HTS machines shows great advantages on high power density, compact structure and lightweight, thus is a promising candidate for wind 
generator and future electrical aircraft propulsion [7-11]. However, REBCO coils have always suffered the problem of low thermal stability and quench damages $[12,13]$. Quench has a significant influence on the safety and reliability of HTS machines, which has to be solved. Conventional quench detection and protection methods applied on low temperature superconductor (LTS) is not suitable for REBCO coils because of its low quench propagation[13-17]. Then, a novel no-insulation (NI) coil technique was proposed for high field magnets to enhance the thermal stability of REBCO coils[18-20]. The key idea of NI coil is to remove the turn-to-turn electrical insulation of traditional insulated coils. Transport current can bypass the local hot spot through turn-to-turn metallic contacts, so that the quench propagation is prevented and an enhanced thermal stability is achieved, which has been validated by both experiments and simulations [21-25]. Since the NI HTS technique has been widely applied on high field magnets so far[26], there is a great potential that it can also be used in HTS motors/generators to improve the thermal stability and prevent quench damage [27].

HTS motors/generators often take the synchronous half-superconducting design to minimize the AC losses, in which the HTS is only applied on rotors windings and the stator windings still use copper wires. In synchronous machines, rotation speed of fundamental fields generated by stator windings is same with the rotation speed of rotor, thus the HTS rotor windings serve as a DC magnet during operations [28-30]. Besides the fundamental fields, the ripple fields from stators are not synchronous to the rotor windings. AC loss of HTS coils induced by the ripple fields has always been a critical issue in the design of conventional HTS machines using insulated HTS coils, since it has a considerable influence on the efficiency of HTS machines[31]. If NI HTS coils was applied on rotors of machines, the ripple field may induce an additional loss on the turn-to-turn contacts, which is called turn-to-turn loss [32, 33]. The AC loss of insulated HTS coils under background AC fields has been studied in detail [31, 34-37]. However, the underlying physics of the AC loss of NI REBCO coils under AC fields are has not been studied thoroughly to help quantify AC losses of NI coils in HTS machine environments, which may be a great challenge for the efficiency of HTS machines with NI technique.

This work aims to investigate the influence of ripple magnetic fields in machines on the HTS NI coils experimentally and numerically, in order to quantify the AC losses. Two NI RECBO coils are wound with different turn-to-turn resistivity, and an insulated HTS coil is also wound as a comparison. Then the AC losses of the three HTS coils exposed to AC external magnetic fields are measured by calibration free methods. Two identify copper magnets are wound for the measurements, which are also used to generate the external magnetic fields. Numerical models are developed to calculate the distribution of induced eddy current in NI coils. Discussion and analysis will be conducted on the results.

\section{Experimental setup}


As shown in Fig.1, two NI HTS coils with different turn-to-turn resistivity are wound by different REBCO tapes, and an insulated HTS coil is also wound for comparison. The NI coil A is wound by tape from SuNAM, South Korea; the NI coil B are wound by tape from Shanghai Superconductor Technology Company Ltd (SSTC), China. Both NI coils have a single pancake (SP) geometry, and two copper sheets are used as current leads. The insulated HTS coil is a double pancake wound by REBCO tape from Superpower, USA wrapped by Kapton. More details of the three HTS coils are shown in Table 1.

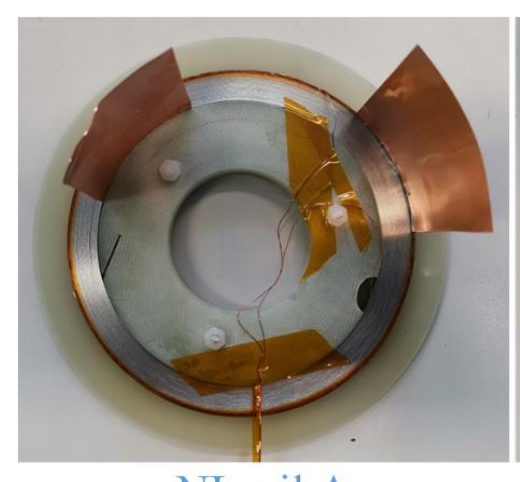

NI coil A

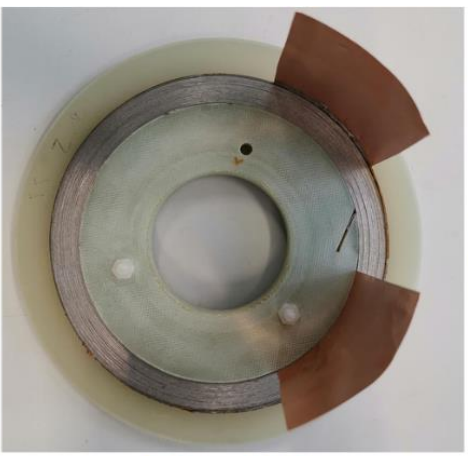

NI coil B

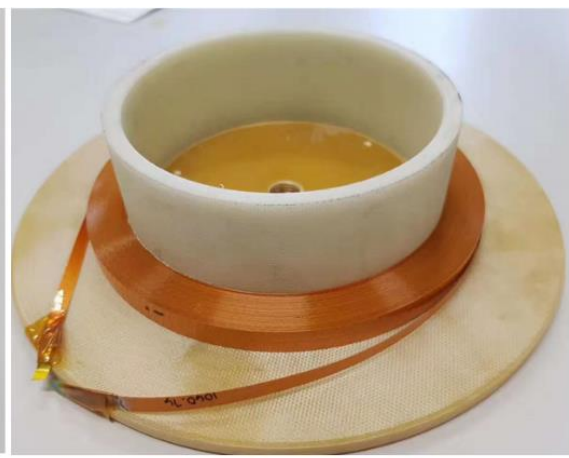

Insulated coil

FIG. 1. The NI HTS coils and insulated HTS coils wound for experiments.

TABLE I. Specification of the test HTS coil and Copper coil.

\begin{tabular}{ccccc}
\hline \hline Parameters & NI coil A & NI coil B & Insulated coil & Copper coil \\
\hline Coil type & $\mathrm{SP}$ & $\mathrm{SP}$ & $\mathrm{DP}$ & Solenoid \\
Inner/outer diameter & $100 / 121 \mathrm{~mm}$ & $100 / 119 \mathrm{~mm}$ & $100 / 126 \mathrm{~mm}$ & $215 / 235 \mathrm{~mm}$ \\
Height & $4.0 \mathrm{~mm}$ & $4.75 \mathrm{~mm}$ & $8.1 \mathrm{~mm}$ & $110 \mathrm{~mm}$ \\
Number of turns & 42 & 38 & $60 * 2$ & 270 \\
Critical current, @77 K & $130 \mathrm{~A}$ & $51 \mathrm{~A}$ & $65 \mathrm{~A}$ & $/$ \\
Inductance & $357 \mu \mathrm{H}$ & $290 \mu \mathrm{H}$ & $2.71 \mathrm{mH}$ & $16.3 \mathrm{mH}$ \\
Filed per Ampere & $478 \mu \mathrm{T} / \mathrm{A}$ & $433 \mu \mathrm{T} / \mathrm{A}$ & $1.37 \mathrm{mT} / \mathrm{A}$ & $1.33 \mathrm{mT} / \mathrm{A}$ \\
Tape producer & $\mathrm{SuNAM}$ & $\mathrm{SSTC}$ & $\mathrm{Superpower}$ & $/$ \\
Width/thickness of tape & $4.0 / 0.25 \mathrm{~mm}$ & $4.75 / 0.25 \mathrm{~mm}$ & $4.0 / 0.15 \mathrm{~mm}$ & $/$ \\
Time constant & $3.0 \mathrm{~s}$ & $1.1 \mathrm{~s}$ & $/$ & $/$ \\
Turn-to-turn resistivity & $39 \mu \Omega \cdot \mathrm{cm}^{2}$ & $112 \mu \Omega \cdot \mathrm{cm}^{2}$ & $/$ & $/$ \\
\hline \hline
\end{tabular}

The AC losses of the three HTS coils exposed to AC external magnetic fields are measured by the calibration free method[38]. Two identical energizing solenoid coils are wound by copper wires, and their details are shown in Table I. One copper coil is used to generate the AC magnetic field acting on the HTS coil (either NI one or the insulated one) placed in its middle plane, as shown in Figure2. The other copper coil is left empty, and both coils are connected in series to the power supply. The AC loss of the HTS coils is absorbed from the power supply energizing the copper coils, in which HTS coils works like an open secondary winding of a transformer (copper coil 
is the primary winding). Each copper coil has a pair of voltage taps and the inductive voltage and resistive loss of copper coils is eliminated by using the bridge concept. Then the AC loss of HTS coil can be obtained by measuring the voltage and current on copper coils without necessity to introduce pick-up coils. More details of the calibration free measurement method can be found in reference[38,39]. Notice that the NI \& insulated HTS coils do not carry a transport current, and they are open circuited during the measurement of $A C$ loss. The copper leads have been removed before measurement to eliminate the eddy loss of copper leads.

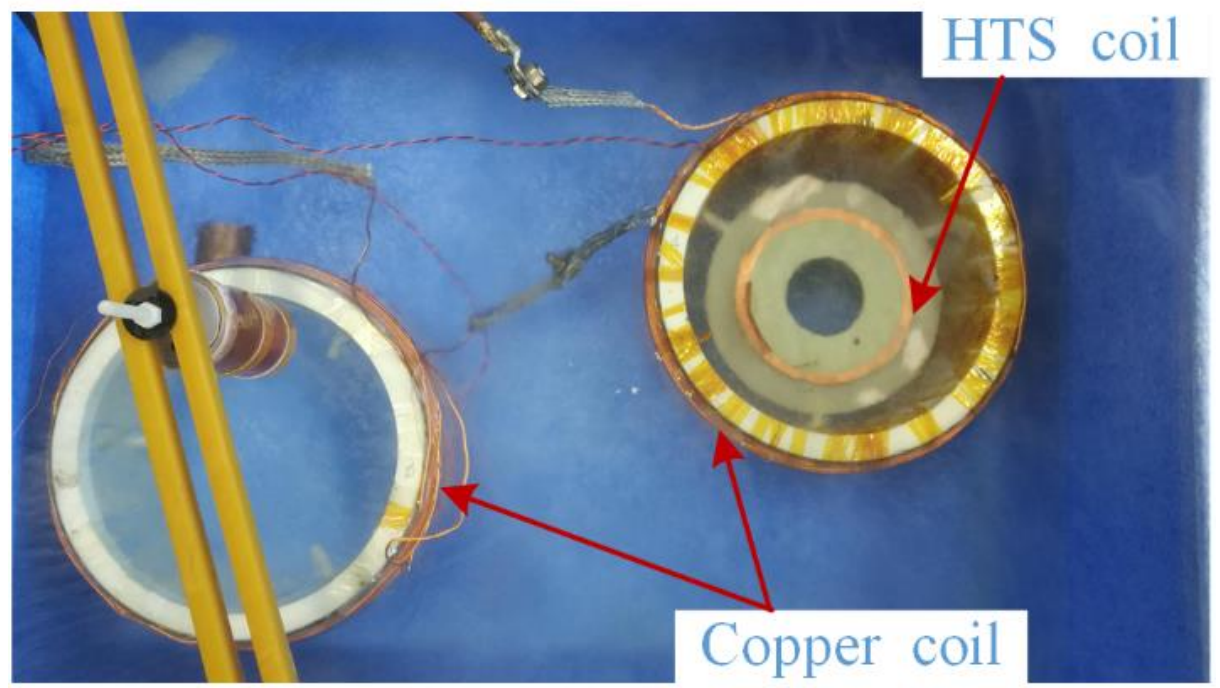

FIG. 2. The calibration free experiment setup for the AC loss measurement of HTS coil exposed to external AC magnetic fields.

\section{Results and discussion}

\section{Turn-to-turn resistivity}

To obtain the turn-to-turn resistivity, fast discharge test are performed on the two NI HTS coils. During the fast discharge test, the $\mathrm{NI}$ coil is first ramped to a transport current below critical current and kept at this current for the time long enough to eliminate the charging delay. Then the current is switched off by an air circuit breaker, and all the magnetic energy stored in the coil is dissipated in the coil itself. A Hall sensor is placed at the center of the coil to measure the magnetic field. Figure 3 shows the field decay in two NI HTS coils after the current is switched off suddenly. Here the initial stable transport current of NI coil A is $80 \mathrm{~A}$ (critical current $130 \mathrm{~A}$ ), that of $\mathrm{NI}$ coil B is $30 \mathrm{~A}$ (critical current $51 \mathrm{~A}$ ). The magnetic field here is normalized by the initial field induced by initial transport current. The field decay matches the following equation[18]:

$$
B=B_{0} e^{-\frac{t}{L / R_{c}}}
$$


where $B$ is the magnetic field measured, $B_{0}$ is the initial magnetic field, $\mathrm{L}$ is inductance of the coil, $R_{c}$ is the equivalent radial resistance. The time constant can be obtained directly from the results in Figure 3 , which is $3.0 \mathrm{~s}$ for $\mathrm{NI}$ coil A, and $1.1 \mathrm{~s}$ for $\mathrm{NI}$ coil B. The equivalent turn-to-turn resistivity $\rho_{t}$ with a unit of $\mu \Omega \cdot \mathrm{cm}^{2}$ can be calculated by[40]:

$$
\rho_{t}=R_{c} / \sum_{k=1}^{N_{t}} \frac{1}{2 \pi r_{k} w_{d}}
$$

where $N_{t}$ is the total number of turns, $r_{k}$ is the radius of the $k$-th turn, $w_{d}$ is the width of the tape. The field of NI coil B drops much faster than NI coil B, the equivalent turn-to-turn resistivity of $\mathrm{NI}$ coil B is $112 \mu \Omega \cdot \mathrm{cm}^{2}$, that of $\mathrm{NI}$ coil A is $39 \mu \Omega \cdot \mathrm{cm}^{2}$.

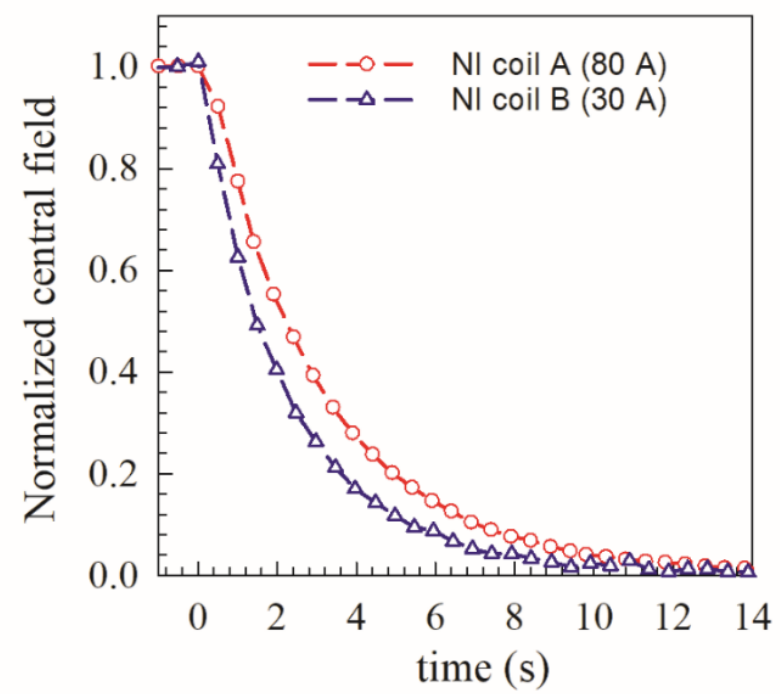

FIG. 3. The decay of normalized magnetic field at the center of two NI HTS coils during the fast discharging test.

\section{AC loss of NI coils induced by ripple magnetic fields}

Both the copper coils and HTS coils are immersed in liquid nitrogen during the AC loss measurement. The copper coils are energized by an AC transport current with frequency of $72 \mathrm{~Hz}$. The results obtained for three HTS coils are shown in Figure 4. As the applied magnetic field is reported that registered at the center of the empty copper coil. Thus we disregard the fact that the presence of HTS coils, especially the NI coils, will have some field shielding effect on the magnetic fields. The results show that the AC losses of both insulated and NI HTS coils increase rapidly with the increase of AC field. Under the same field amplitude the AC loss of the NI HTS coils can be more than 20 times of that in the insulated HTS coil. For the insulated HTS coil with an open circuit, the AC loss is the magnetization loss in superconducting layers. This is mainly induced by the perpendicular component of the 
AC field expected due to the fact that the position of HTS coils in copper coils is slightly higher than the middle plane.

The AC losses in NI HTS coils consist of three parts: the magnetization loss in superconducting layers, the Joule loss in turn-to-turn contacts, and the transport loss in superconducting layers. Due to the absence of insulation, a closed circuit is formed through the turn-to-turn contacts, so an eddy current is induced by the applied AC field. Part of this induced AC eddy current flows along the azimuthal direction, and thus generates an additional transport loss in superconducting layers that would not exist in insulated HTS coils at the circumstances of our experiment. Meanwhile, the induced eddy current also generates Joule loss on the turn-to-turn contact resistance, which is called turn-to-turn loss in this study. The induced eddy current should have a field shielding effect on the HTS tapes, so the magnetization loss of superconducting layers in NI HTS coils should be lower than that in its insulated counterpart. The measurements in Figure 4 shows that the turn-to-turn loss is overwhelming majority of total AC losses in NI HTS coils, and the other losses (transport loss and magnetization loss) in superconducting layers are negligible. As a result, the NI HTS coils generate much more AC losses than the insulated coil.

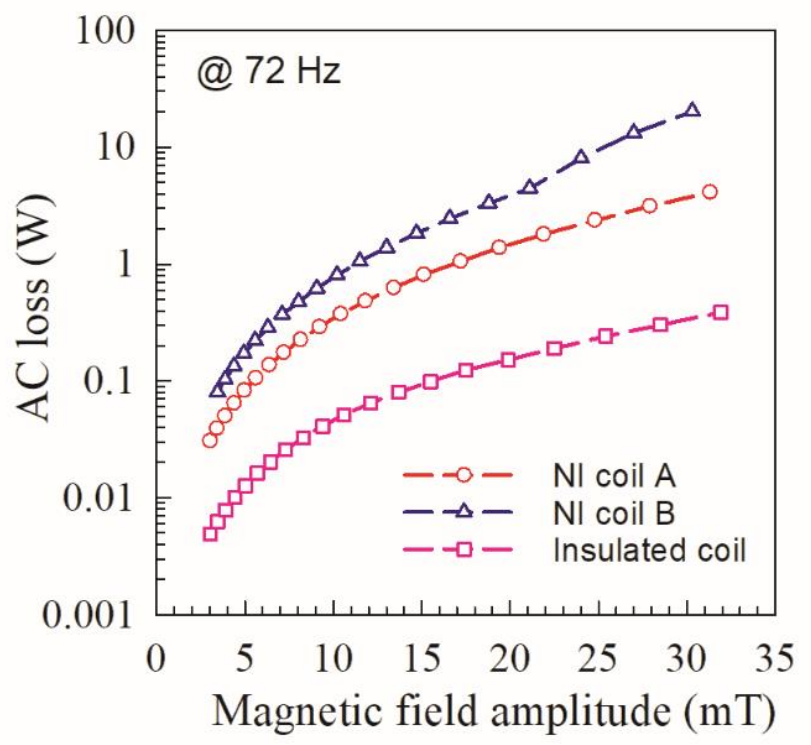

FIG. 4. The AC loss of HTS coils exposed to external AC magnetic fields.

The results in Figure 4 also shows that NI coil A with a turn-to-turn resistivity of $39 \mu \Omega \cdot \mathrm{cm}^{2}$ generates much less AC loss than the $\mathrm{NI}$ coil B with a turn-to-turn resistivity of $112 \mu \Omega \cdot \mathrm{cm}^{2}$. The turn-to-turn loss $W_{\mathrm{t} 2 \mathrm{t}}$ of $\mathrm{NI}$ coils can be calculated by:

$$
W_{t 2 t}=\sum_{k=1}^{N_{t}} I_{k}^{2} R_{k}
$$


where $R_{k}$ is the turn-to-turn resistance of the $k$-turn, and $l_{k}$ is the induced eddy current flowing though the turnto-turn contact of the $k$-th turn. Lower turn-to-turn resistivity leads to higher eddy current. Since the turn-to-turn loss is the product of turn-to-turn contact resistance and the square of induced eddy current, lower turn-to-turn resistivity does not always lead to higher turn-to-turn losses. If the turn-to-turn resistivity drops to zero, the winding of NI HTS coil will be shielded from the external AC fields, and the AC loss will be reduced. On the other hand if the turn-to-turn resistivity is infinite, the NI HTS coil will change to an insulated HTS coil, thus only the superconductor magnetization loss is induced, which is much lower than AC loss of NI HTS coils. In other words we expect the behavior typical for eddy current loss in metals, where the decrease of resistivity increases the loss first reaching a peak point, and afterwards causes it to drop again. Obviously this peak point should be avoided when the NI HTS coil is applied in ripple fields.

Therefore, the AC loss of NI HTS coils exposed to AC external magnetic fields can be reduced by both increasing and reducing the turn-to-turn resistivity. Lowering turn-to-turn resistivity always means better thermal stability, while this will lead to more serious charging delay, which is also challenging for the fast magnetization and demagnetization of NI HTS coils [40, 41]. Increasing the turn-to-turn resistivity can significantly reduce the charging delay, while this may also considerably reduce the thermal stability of NI HTS coils. Therefore, an optimal design on the turn-to-turn resistivity is required on the NI HTS coils when it is applied on machines.

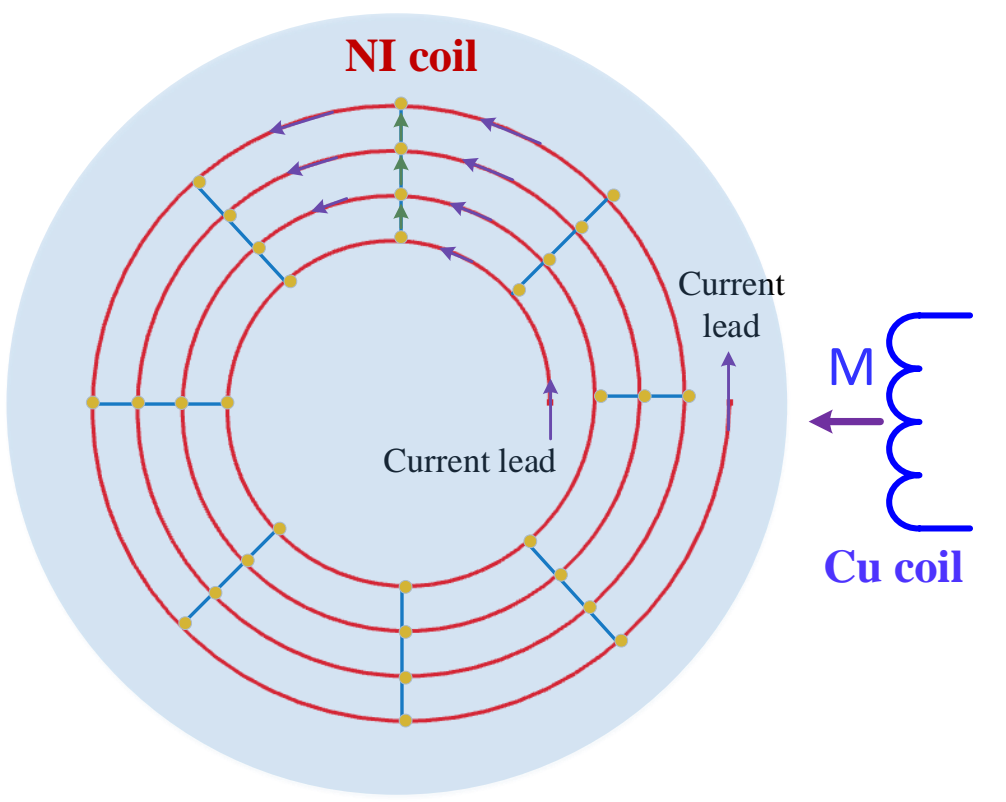

FIG. 5. The schematic illustration of the equivalent circuit network model for NI HTS coils exposed to external magnetic fields. Notice that each turn is subdivided to 8 elements in this figure, which is for clear presentation. In the calculation of this study, each turn is subdivide 48 arc elements. 


\section{Distribution of induced eddy current}

An equivalent circuit model is developed to study the distribution of the induced eddy current as well as turn-toturn loss on the NI HTS coil. In this model, the eddy current is decomposed to the azimuthal component $i$ and radial component $j$. Each turn of the coil is subdivided to fine arc element, and each element is equivalent to lumped circuit model. The whole coil is equivalent to a distributed circuit network [42-46], as shown in Figure 5. The external magnetic fields induced by copper coils are represented by coupling through the mutual inductance $M$. The governing equation of this model can be derived from Kirchhoff laws:

$$
\left\{\begin{array}{l}
\sum_{k} i_{k}+\sum_{k} j_{k}=0 \\
\sum_{m=1}^{4} u_{m, k}=0
\end{array}\right.
$$

where $u_{m, k}$ is the voltage on the $m$-th circuit branch of the $k$-th independent circuit mesh. More details about this model have been introduced in references $[47,48]$.

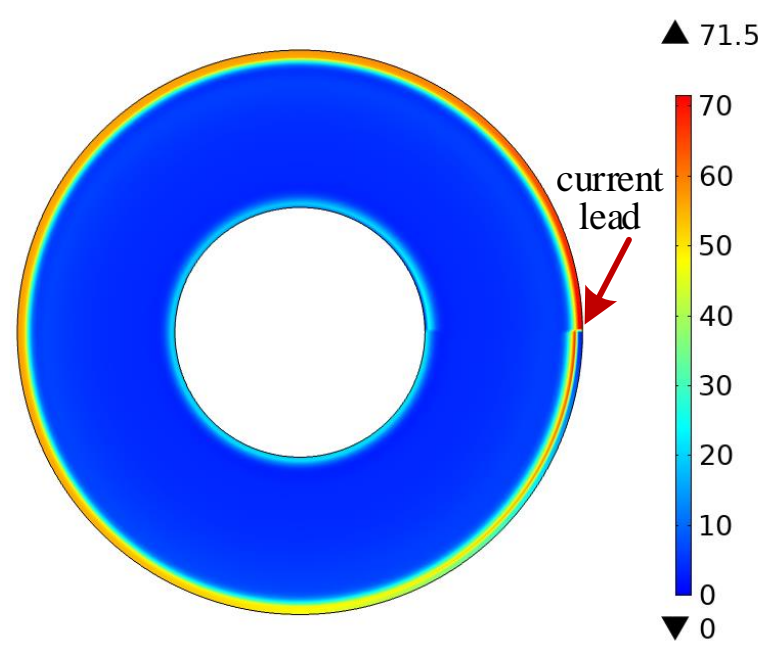

(a) azimuthal current (A)

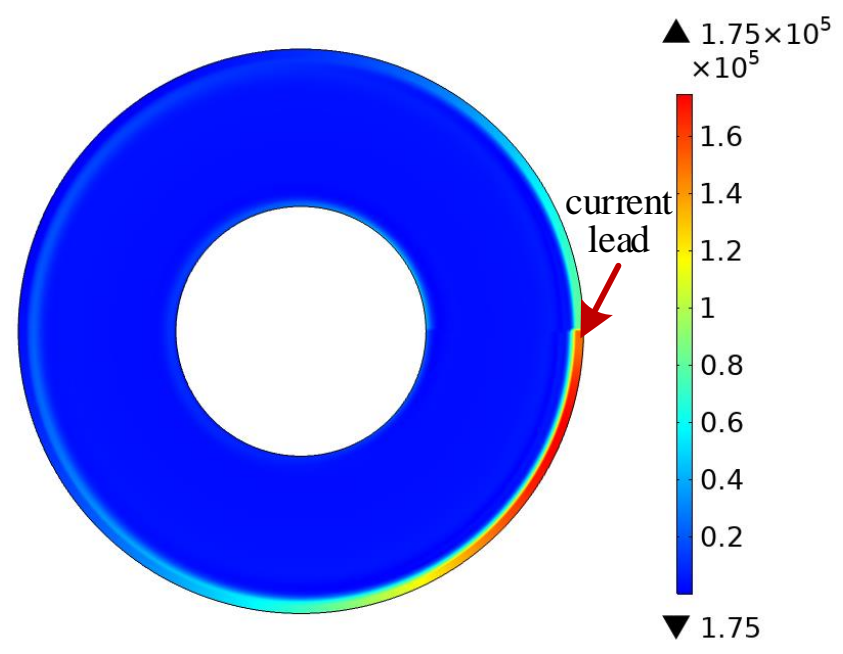

(b) radial current density $\left(\mathrm{A} / \mathrm{m}^{2}\right)$

FIG. 6. The amplitude of the induced eddy current on NI HTS coil A, the background field generated by cooper coil is 5.6 $\mathrm{mT} / 72 \mathrm{~Hz}$, turn-to-turn resistivity $39 \mu \Omega \cdot \mathrm{cm}^{2}$. 


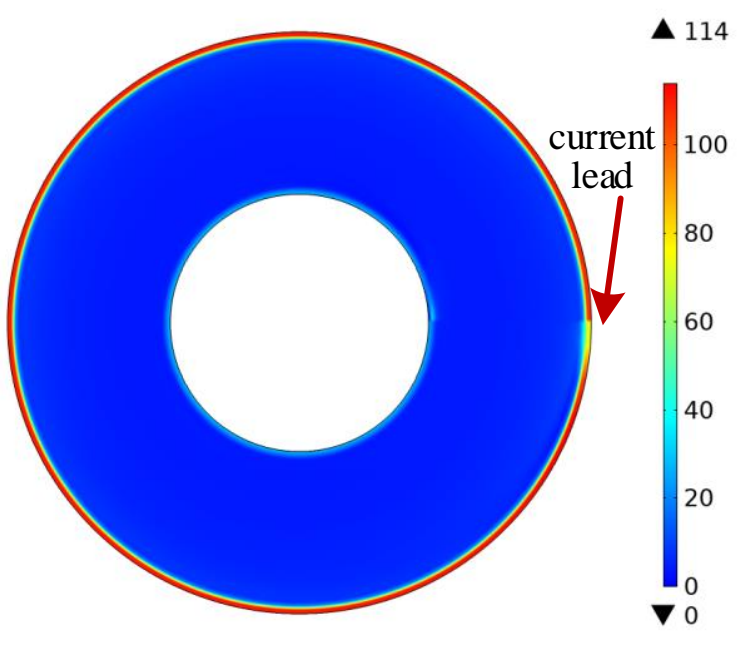

(a) azimuthal current (A)

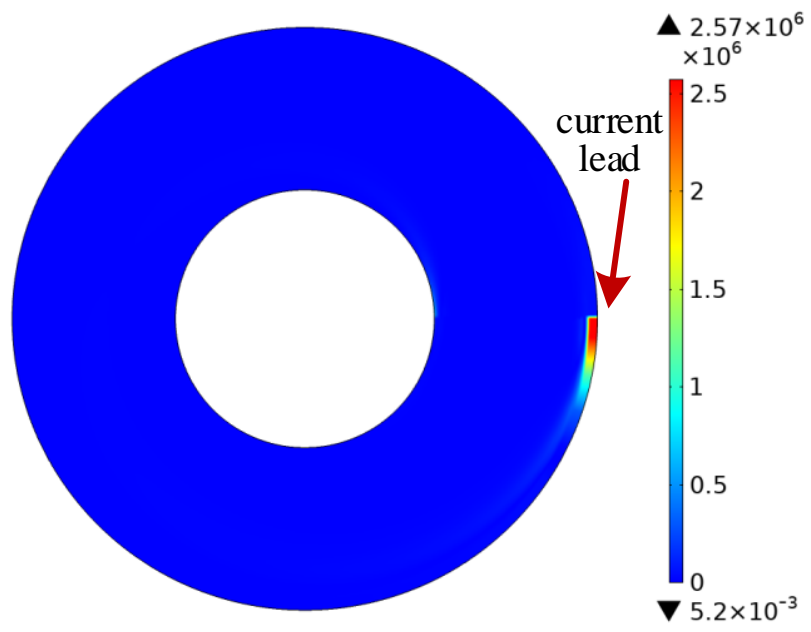

(b) radial current density $\left(\mathrm{A} / \mathrm{m}^{2}\right)$

FIG. 7. The amplitude of the induced eddy current on NI HTS coil A, the background field generated by cooper coil is 5.6 $\mathrm{mT} / 72 \mathrm{~Hz}$, turn-to-turn resistivity $1 \mu \Omega \cdot \mathrm{cm}^{2}$.

Figure 6 shows the distribution of induced eddy current in NI HTS coil A. Notice that the thickness of each turn is enlarged 30 times for better presentation. We observe kind of skin effect, when most of eddy current is generated on the inner and outer turns of the NI HTS coil, especially the outer turns. That's because the outer turns have relatively larger diameter, thus couples more external magnetic flux than other turns. Then the induced eddy current has a field shielding effects on the other turns, so that no current is induced on these turns. The azimuthal current (transport current induced) flows in superconducting layers, and the closed circuit is formed through the turn-to-turn contacts, which leads the radial current. A very small AC field $(5.6 \mathrm{mT} / 72 \mathrm{~Hz}$ in Figure 6) can induce a very high transport current (up to 71.5 A in Figure 6) in the HTS tapes, while the rest of turns do not carry any eddy current. This will add an extra overcurrent quench risk on the outer turns during operation, which has to be eliminated.

The turn-to-turn resistivity of NI HTS coils ranges from $1 \mu \Omega \cdot \mathrm{cm}^{2}$ to $200 \mu \Omega \cdot \mathrm{cm}^{2}$ in Liquid nitrogen [49-51]. We change the turn-to-turn resistivity of $\mathrm{NI}$ coil A to $1 \mu \Omega \cdot \mathrm{cm}^{2}$, re-calculate the model and obtain the distribution of eddy current induced by magnetic fields $5.6 \mathrm{mT} / 72 \mathrm{~Hz}$, as shown in Figure 7. With lower turn-to-turn resistivity, a more significant skin effect is observed, and a higher azimuthal current (transport current) is induced in outer turns of the NI coil. This will increase the quench risk of the NI HTS coil, although lower turn-to-turn resistivity generates less turn-to-turn loss. With a relatively higher turn-to-turn resistivity, the radial current has more uniform distribution along the angular direction of the coil, as shown in Figure 6(b). With low turn-to-turn resistivity, most of induced eddy current flows in superconducting layers along the azimuthal current, and the current path closes at the connecting position of the outermost turn and its adjacent turn, thus the radial current, 
as well as the turn-to-turn loss, shows a significant accumulation at this point, as shown in Figure $7(b)$. This may induce a local hot spot and thus increases the quench risk to some extent.

\section{Conclusion}

To sum up, we measured the AC loss of NI HTS coils exposed to AC external magnetic fields and compared it with insulated HTS coils. The results show that that our NI HTS coils generate more than 20 times higher AC loss than insulated HTS coil. Therefore, AC loss may be challenging for the efficiency of HTS machines, when the NI coil is applied in machine environment with ripple fields, though the $\mathrm{NI}$ technique can significantly enhance the stability and reliability of the HTS windings. Experiments and theoretical consideration predict that the AC loss of NI HTS coils induced by ripple fields can be significantly reduced by both decreasing the turn-to-turn resistivity and increasing the turn-to-turn resistivity. Therefore, an optimization of turn-to-turn resistivity is required when the $\mathrm{NI}$ technique is applied on the HTS machines. Most of eddy current is induced in the outer turns of the NI HTS coil, and a very small external ripple field may induce a very high eddy current on outer turns. Lower turn-to-turn resistivity leads to more significant accumulation of radial current as well as turn-to-turn loss. These may lead to a quench risk, which needs a special attention in coil design.

\section{ACKNOWLEDGMENTS}

This project was supported European Union's Horizon 2020 research and innovation programme under the Marie Sklodowska-Curie grant agreement No 799902 and by the Grant Agency VEGA under Contract 1/0151/17.

\section{REFERENCES}

[1] S. Samoilenkov et al., "Customised 2G HTS wire for applications," Superconductor Science \& Technology, vol. 29, no. 2, Feb 2016, Art. no. 024001.

[2] Y. Zhao et al., "Progress in fabrication of second generation high temperature superconducting tape at Shanghai Superconductor Technology," Superconductor Science and Technology, vol. 32, no. 4, p. 044004, 2019/02/25 2019.

[3] S. Hahn et al., "Operation and performance analyses of 350 and $700 \mathrm{MHz}$ low-/high-temperature superconductor nuclear magnetic resonance magnets: A march toward operating frequencies above $1 \mathrm{GHz}, "$ Journal of Applied Physics, vol. 105, no. 2, Jan 2009, Art. no. 024501.

[4] T. Murakami and Y. Okuno, "Experiments and numerical simulations on high-density magnetohydrodynamic electrical power generation," Journal of Applied Physics, vol. 104, no. 6, Sep 2008, Art. no. 063307.

[5] W. Yawei, Z. Min, G. Francesco, Z. Zixuan, and Y. Weijia, "Study of the magnetization loss of CORC ${ }^{\circledR}$ cables using a 3D T-A formulation," Superconductor Science and Technology, vol. 32, no. 2, p. 025003, 2019.

[6] J. W. Li, Q. Q. Yang, F. Robinson, F. Liang, M. Zhang, and W. J. Yuan, "Design and test of a new droop control algorithm for a SMES/battery hybrid energy storage system," Energy, vol. 118, pp. 1110-1122, Jan 2017.

[7] B. Sarlioglu and C. T. Morris, "More Electric Aircraft: Review, Challenges, and Opportunities for Commercial Transport Aircraft," IEEE Transactions on Transportation Electrification, vol. 1, no. 1, pp. 54-64, 2015.

[8] C. E. Jones, P. J. Norman, S. J. Galloway, M. J. Armstrong, and A. M. Bollman, "Comparison of Candidate Architectures for Future Distributed Propulsion Aircraft," Ieee Transactions on Applied Superconductivity, vol. 26, no. 6, Sep 2016, Art. no. 3601409.

[9] M. Zhang, F. Eastham, and W. J. Yuan, "Design and Modeling of 2G HTS Armature Winding for Electric Aircraft Propulsion Applications," Ieee Transactions on Applied Superconductivity, vol. 26, no. 3, Apr 2016, Art. no. 5205705. 
[10] F. Berg, J. Palmer, P. Miller, and G. Dodds, "HTS System and Component Targets for a Distributed Aircraft Propulsion System," Ieee Transactions on Applied Superconductivity, vol. 27, no. 4, Jun 2017, Art. no. 3600307.

[11] X. Song, Y. Wang, D. Liu, S. Wang, and D. Liang, "Short-Circuit Characteristics of a High Temperature Superconducting Wind Turbine Generator Employing a Segmented Armature Winding," IEEE Transactions on Applied Superconductivity, vol. 29, no. 5, pp. 1-5, 2019.

[12] M. Oomen, W. Herkert, D. Bayer, P. Kummeth, W. Nick, and T. Arndt, "Manufacturing and test of 2G-HTS coils for rotating machines: Challenges, conductor requirements, realization," (in English), Physica C-Superconductivity and Its Applications, Article vol. 482, pp. 111-118, Nov 2012.

[13] H. H. Song, K. Gagnon, and J. Schwartz, "Quench behavior of conduction-cooled YBa2Cu3O7-delta coated conductor pancake coils stabilized with brass or copper," (in English), Superconductor Science \& Technology, Article vol. 23, no. 6, p. 10, Jun 2010, Art. no. 065021.

[14] S. Tengming, Y. Liyang, T. Daniele, and L. Pei, "High-field quench behavior and dependence of hot spot temperature on quench detection voltage threshold in a $\mathrm{Bi} 2 \mathrm{Sr} 2 \mathrm{CaCu} 2 \mathrm{O}$ x coil," Superconductor Science and Technology, vol. 28, no. 7, p. 075014, 2015.

[15] Y. Liyang, L. Pei, S. Tengming, and S. Justin, "Quench degradation limit of multifilamentary Ag/Bi $2 \mathrm{Sr} 2 \mathrm{CaCu} 2 \mathrm{O}$ x round wires," Superconductor Science and Technology, vol. 29, no. 3, p. 035010, 2016.

[16] Y. Wang, J. Zheng, Z. Zhu, M. Zhang, and W. Yuan, "Quench behavior of high-temperature superconductor (RE)Ba2Cu3O x CORC cable," Journal of Physics D: Applied Physics, vol. 52, no. 34, p. 345303, 2019/06/24 2019.

[17] H. Song and J. Schwartz, "Stability and Quench Behavior of YBa2Cu3O7-x Coated Conductor at 4.2 K, Self-Field," Ieee Transactions on Applied Superconductivity, vol. 19, no. 5, pp. 3735-3743, Oct 2009.

[18] S. Hahn, D. K. Park, J. Bascunan, and Y. Iwasa, "HTS Pancake Coils Without Turn-to-Turn Insulation," (in English), Ieee Transactions on Applied Superconductivity, Article vol. 21, no. 3, pp. 1592-1595, Jun 2011.

[19] S. Hahn et al., "No-insulation multi-width winding technique for high temperature superconducting magnet," Applied Physics Letters, vol. 103, no. 17, Oct 21 2013, Art. no. 173511.

[20] Y. Iwasa and S. Hahn, "First-cut design of an all-superconducting 100-T direct current magnet," Applied Physics Letters, vol. 103, no. 25, Dec 16 2013, Art. no. 253507.

[21] Y. H. Choi et al., "Thermal Quench Behaviors of No-Insulation Coils Wound Using GdBCO Coated Conductor Tapes With Various Lamination Materials," (in English), Ieee Transactions on Applied Superconductivity, Article vol. 24, no. 3, p. 5, Jun 2014, Art. no. 8800105.

[22] J.-B. Song, S. Hahn, T. Lecrevisse, J. Voccio, J. Bascunan, and Y. Iwasa, "Over-current quench test and self-protecting behavior of a 7 T/78 mm multi-width no-insulation REBCO magnet at 4.2 K," Superconductor Science \& Technology, vol. 28, no. 11, Nov 2015, Art. no. 114001.

[23] W. D. Markiewicz, J. J. Jaroszynski, D. V. Abraimov, R. E. Joyner, and A. Khan, "Quench analysis of pancake wound REBCO coils with low resistance between turns," Superconductor Science \& Technology, vol. 29, no. 2, pp. 25001-25001, Feb 2016, Art. no. 025001 .

[24] T. M. Qu, P. C. Michael, J. Voccio, J. Bascunan, S. Hahn, and Y. Iwasa, "Persistent-current switch for pancake coils of rare earthbarium-copper-oxide high-temperature superconductor: Design and test results of a double-pancake coil operated in liquid nitrogen (77-65 K) and in solid nitrogen (60-57 K)," Applied Physics Letters, vol. 109, no. 8, Aug 2016, Art. no. 082601.

[25] W. K. Chan and J. Schwartz, "Improved stability, magnetic field preservation and recovery speed in (RE)Ba2Cu3Ox-based noinsulation magnets via a graded-resistance approach," Superconductor Science and Technology, Article vol. 30, no. 7, 2017, Art. no. 074007.

[26] S. Hahn et al., "45.5-tesla direct-current magnetic field generated with a high-temperature superconducting magnet," Nature, 2019/06/12 2019.

[27] U. Bong et al., "A Design Study on 40 MW Synchronous Motor With No-Insulation HTS Field Winding," IEEE Transactions on Applied Superconductivity, vol. 29, no. 5, pp. 1-6, 2019.

[28] M. Furuse, M. Yoshikawa, Y. Itoh, S. Fukui, and T. Nakamura, "Fabrication and Testing of Racetrack-Shaped Double-Pancake Coil for Stator Windings of Induction-Synchronous Motor," IEEE Transactions on Applied Superconductivity, vol. 25, no. 3, pp. 1-4, 2015.

[29] P. Song, T. M. Qu, L. F. Lai, M. S. Wu, X. Y. Yu, and Z. Han, "Thermal analysis for the HTS stator consisting of HTS armature windings and an iron core for a 2.5 kW HTS generator," Superconductor Science and Technology, vol. 29, no. 5, p. $054007,2016$.

[30] M. Heejong, K. Yeong-Chun, P. Heui-Joo, Y. In-Keun, and P. Minwon, "An introduction to the design and fabrication progress of a megawatt class 2G HTS motor for the ship propulsion application," Superconductor Science and Technology, vol. 29, no. 3, p. 034009,2016

[31] M. Zhang, W. Yuan, J. Kvitkovic, and S. Pamidi, "Total AC loss study of 2G HTS coils for fully HTS machine applications," Superconductor Science \& Technology, vol. 28, no. 11, Nov 2015, Art. no. 115011.

[32] Y. Wang, H. Song, W. Yuan, Z. Jin, and Z. Hong, "Ramping turn-to-turn loss and magnetization loss of a No-Insulation (RE) Ba2Cu3Ox high temperature superconductor pancake coil," Journal of Applied Physics, vol. 121, no. 11, Mar 2017, Art. no. 113903.

[33] Y. W. Wang, M. Zhang, W. J. Yuan, Z. Y. Hong, Z. J. Jin, and H. H. Song, "Non-uniform ramping losses and thermal optimization with turn-to-turn resistivity grading in a (RE) Ba2Cu3Ox magnet consisting of multiple no-insulation pancake coils," Journal of Applied Physics, vol. 122, no. 5, Aug 2017, Art. no. 053902.

[34] M. Furuse, J. Kondoh, H. Tanaka, and M. Umeda, "AC loss measurement of HTS coils with ferromagnetic disks," IEEE Transactions on Applied Superconductivity, vol. 13, no. 2, pp. 2356-2359, 2003. 
[35] M. D. Ainslie, W. Yuan, and T. J. Flack, "Numerical Analysis of AC Loss Reduction in HTS Superconducting Coils Using Magnetic Materials to Divert Flux," IEEE Transactions on Applied Superconductivity, vol. 23, no. 3, pp. 4700104-4700104, 2013.

[36] M. D. Ainslie, V. M. Rodriguez-Zermeno, Z. Hong, W. Yuan, T. J. Flack, and T. A. Coombs, "An improved FEM model for computing transport AC loss in coils made of RABiTS YBCO coated conductors for electric machines," Superconductor Science and Technology, vol. 24, no. 4, p. 045005, 2011/01/27 2011.

[37] V. Lahtinen, E. Pardo, J. Souc, M. Solovyov, and A. Stenvall, "Ripple field losses in direct current biased superconductors: Simulations and comparison with measurements," Journal of Applied Physics, vol. 115, no. 11, Mar 2014, Art. no. 113907.

[38] J. Šouc, F. Gömöry, and M. Vojenčiak, "Calibration free method for measurement of the AC magnetization loss," Superconductor Science and Technology, vol. 18, no. 5, pp. 592-595, 2005/03/16 2005.

[39] J. Šouc et al., "Low AC loss cable produced from transposed striated CC tapes," Superconductor Science and Technology, vol. 26, no. 7, p. 075020, 2013.

[40] Y. Wang and H. Song, "Influence of turn-to-turn resistivity and coil geometrical size on charging characteristics of no-electricalinsulation REBCO pancake coils," Superconductor Science and Technology, vol. 29, no. 7, p. 075006, 2016.

[41] Y. Wang et al., "Analysis and Comparison Between No-Insulation and Metallic Insulation REBCO Magnet for the Engineering Design of a 1-MW DC Induction Heater," IEEE Transactions on Applied Superconductivity, vol. 27, no. 4, pp. 1-5, 2017.

[42] H. L. Quach et al., "Analysis on Electrical and Thermal Characteristics of a No-Insulation HTS Coil Considering Heat Generation in Steady and Transient States," IEEE Transactions on Applied Superconductivity, vol. 29, no. 5, pp. 1-6, 2019.

[43] M. Cho et al., "Combined Circuit Model to Simulate Post-Quench Behaviors of No-Insulation HTS Coil," IEEE Transactions on Applied Superconductivity, vol. 29, no. 5, pp. 1-5, 2019.

[44] S. Noguchi, R. Itoh, S. Hahn, and Y. Iwasa, "Numerical Simulation of Superconducting Coil Wound With No-Insulation NbTi Wire," Ieee Transactions on Applied Superconductivity, vol. 24, no. 3, Jun 2014, Art. no. 4900504.

[45] L. Donghui, Z. Weiwei, Y. Huadong, and Z. Youhe, "Thermal stability and mechanical behavior in no-insulation high-temperature superconducting pancake coils," Superconductor Science and Technology, vol. 31, no. 8, p. 085010, 2018.

[46] D. Liu, W. Zhang, H. Yong, and Y. Zhou, "Numerical analysis of thermal stability and mechanical response in a no-insulation hightemperature superconducting layer-wound coil," Superconductor Science and Technology, vol. 32, no. 4, p. 044001, 2019/02/15 2019.

[47] Y. Wang, H. Song, D. Xu, Z. Y. Li, Z. Jin, and Z. Hong, "An equivalent circuit grid model for no-insulation HTS pancake coils," Superconductor Science \& Technology, vol. 28, no. 4, Apr 2015, Art. no. 045017.

[48] Y. Wang, C. Wan Kan, and J. Schwartz, "Self-protection mechanisms in no-insulation (RE)Ba $2 \mathrm{Cu} 3 \mathrm{O} x$ high temperature superconductor pancake coils," Superconductor Science and Technology, vol. 29, no. 4, pp. 045007 (11 pp.)-045007 (11 pp.), April 2016.

[49] M. Bonura, C. Barth, A. Joudrier, J. F. Troitino, A. Fête, and C. Senatore, "Systematic Study of the Contact Resistance Between REBCO Tapes: Pressure Dependence in the Case of No-Insulation, Metal Co-Winding and Metal-Insulation," IEEE Transactions on Applied Superconductivity, vol. 29, no. 5, pp. 1-5, 2019.

[50] M. Sohn, K. Sim, B. Eom, H. Ha, H. Kim, and K. Seong, "Controllability of the Contact Resistance of 2G HTS Coil With Metal Insulation," IEEE Transactions on Applied Superconductivity, vol. 28, no. 3, pp. 1-5, 2018.

[51] L. Jun, G. Robert, H. Ke, and H. Seungyong, "Contact resistance between two REBCO tapes under load and load cycles," Superconductor Science and Technology, vol. 30, no. 4, p. 045005, 2017. 